\title{
Dynamic Heterogeneity in the Glauber-Ising chain
}

\author{
Peter Mayer ${ }^{\dagger, \ddagger}$, Peter Sollich ${ }^{\ddagger}$, Ludovic Berthier ${ }^{\S, \|}$ and Juan P. \\ Garrahan \\ ${ }^{\dagger}$ Department of Chemistry, Columbia University, 3000 Broadway, New York, NY \\ 10027, US \\ ‡Department of Mathematics, King’s College, Strand, London, WC2R 2LS, UK \\ $\S$ Theoretical Physics, University of Oxford, 1 Keble Road, Oxford, OX1 3NP, UK \\ ||Laboratoire des Colloïdes, Verres et Nanomatériaux, Université Montpellier II and \\ UMR 5587 CNRS, 34095 Montpellier Cedex 5, France \\ `School of Physics and Astronomy, University of Nottingham, Nottingham, NG7 \\ 2RD, UK
}

\begin{abstract}
In a recent paper [P. Mayer et al., Phys. Rev. Lett. 93, 115701 (2004)] it was shown, by means of experiments, theory and simulations, that coarsening systems display dynamic heterogeneity analogous to that of glass formers. Here, we present a detailed analysis of dynamic heterogeneities in the Glauber-Ising chain. We discuss how dynamic heterogeneity in Ising systems must be measured through connected multipoint correlation functions. We show that in the coarsening regime of the Ising chain these multi-point functions reveal the growth of spatial correlations in the dynamics, beyond what can be inferred from standard two-point correlations. They have nontrivial scaling properties, which we interpret in terms of the diffusion-annihilation dynamics of domain walls. In the equilibrium dynamics of the Ising chain, on the other hand, connected multi-point functions vanish exactly and dynamic heterogeneity is not observed. Our results highlight the similarities between coarsening systems and glass formers.
\end{abstract}




\section{Introduction}

An obvious question to ask about glass-forming liquids is whether the dramatic slowdown of the dynamics on cooling is correlated with a corresponding increase in an appropriately defined length scale. Critical slowing-down around second-order phase transitions, for example, is correlated with the divergence of a static correlation length. In supercooled liquids and glasses, the consensus is that there is no growing static length scale, since the static structure - as measured, e.g., by the amplitude of density fluctuations - changes only negligibly while relaxation time scales grow by orders of magnitude. Any growing length scale in glassy systems must therefore reflect the spatial structure of the dynamics. In order for this spatial structure to be non-trivial, the dynamics must vary from point to point: it must be heterogeneous. The simplest conceptual picture of such dynamical heterogeneity is that some regions of a material are fast and others slow. The identities and locations of these regions may of course change over time.

We will not try to review the literature on dynamical heterogeneity, which is vast, and refer instead to [1, 2, 3. We focus in this paper on the characterization of dynamical heterogeneities via multi-point correlations [4, 15, 6, 7, 8, 9, 10, 11, 12, In the context of lattice models, one considers a spatial correlation function between sites $i$ and $j$, of the general form $C_{i j}=\left\langle F_{i} F_{j}\right\rangle-\left\langle F_{i}\right\rangle\left\langle F_{j}\right\rangle$, but with $F_{i}$ itself a twotime quantity such as $F_{i}=A_{i}(t) A_{i}\left(t_{\mathrm{w}}\right)$ and $A$ some local observable. Thus $C_{i j}$ is a four-point correlation function. It essentially measures how the relaxation of $A$ at point $i$ is correlated with the relaxation at point $j$. Such a definition should therefore pick up dynamical heterogeneities. Associated with $C_{i, j}$ is a so-called susceptibility, i.e., the spatially integrated correlation $\chi=(1 / N) \sum_{i j} C_{i j}$ : if the length scale on which the dynamics is correlated grows, then so should this fourth-order susceptibility.

It is worth noting that the four-point susceptibility $\chi=(1 / N) \sum_{i j} C_{i j}$ can be reexpressed as $\chi=(1 / N)\left[\left\langle q^{2}\right\rangle-\langle q\rangle^{2}\right]$ where $q=\sum_{i} A_{i}(t) A_{i}\left(t_{\mathrm{w}}\right)$ measures the "overlap" between configurations at time $t_{\mathrm{w}}$ and $t$. From this we can easily gain qualitative insight into the dependence of $\chi$ on $\Delta t=t-t_{\mathrm{w}}$. At $\Delta t=0$, one does not expect large fluctuations in $q$; indeed, in a spin system and for $A_{i}=\sigma_{i}, q$ is a constant for $t=t_{\mathrm{w}}$ and hence $\chi=0$. If, in the opposite limit $\Delta t \rightarrow \infty$, the system decorrelates from its state at time $t_{\mathrm{w}}$, then one expects $q$ to decay to a small value, again with only minor fluctuations. For intermediate times, however, the fact that a glass may remain trapped near the $t_{\mathrm{w}}$-configuration for a time that is both long and strongly dependent on the state at $t_{\mathrm{w}}$ leads to large fluctuations of $q$ between different dynamical histories and therefore to a large value of $\chi$.

The usefulness of four-point correlations for understanding heterogeneous dynamics motivates us to consider their behaviour in coarsening systems [4]. Because - contrary to glass formers - coarsening systems develop strong spatial correlations at long times, appropriately modified four-point correlations must be considered. It was shown in a recent letter [13] that these reveal non-trivial spatio-temporal correlations in coarsening 
systems which show strong analogies with aging glasses. This is, in fact, also the case for the Glauber-Ising chain, which we study in this paper. Its analytical tractability makes it an interesting candidate for exploring four-point correlations in coarsening dynamics. Results were partially announced in [13.

Explicitly, the standard four-point function that is studied in the literature on dynamical heterogeneities becomes for our spin system

$$
C_{l-k}\left(\Delta t, t_{\mathrm{w}}\right)=\left\langle\sigma_{k}(t) \sigma_{k}\left(t_{\mathrm{w}}\right) \sigma_{l}(t) \sigma_{l}\left(t_{\mathrm{w}}\right)\right\rangle-\left\langle\sigma_{k}(t) \sigma_{k}\left(t_{\mathrm{w}}\right)\right\rangle\left\langle\sigma_{l}(t) \sigma_{l}\left(t_{\mathrm{w}}\right)\right\rangle,
$$

where $t=\Delta t+t_{\mathrm{w}} \geq t_{\mathrm{w}} \geq 0$ and the times $t, t_{\mathrm{w}}$ are measured from the quench. The definition (11) implies that $C_{n}\left(\Delta t, t_{\mathrm{w}}\right)$ is an even function of $n$. At $\Delta t=0$ it in fact vanishes for all $n$ since Ising spins satisfy $\sigma^{2}=1$. In the opposite limit $\Delta t \rightarrow \infty$, because the configurations at $t_{\mathrm{w}}$ and $t$ will decorrelate, $C_{l-k}\left(\Delta t, t_{\mathrm{w}}\right)$ approaches $\left\langle\sigma_{k}\left(t_{\mathrm{w}}\right) \sigma_{l}\left(t_{\mathrm{w}}\right)\right\rangle\left\langle\sigma_{k}(t) \sigma_{l}(t)\right\rangle-\left\langle\sigma_{k}(t)\right\rangle\left\langle\sigma_{k}\left(t_{\mathrm{w}}\right)\right\rangle\left\langle\sigma_{l}(t)\right\rangle\left\langle\sigma_{l}\left(t_{\mathrm{w}}\right)\right\rangle$. The last term vanishes, so this limit reduces to the product of the spatial correlations at times $t_{\mathrm{w}}$ and $t \rightarrow \infty$. This argument holds quite generally in a spin system without an overall magnetization. However, in a typical glassy system the spatial correlations at times $t_{\mathrm{w}}$ and $t$ will be comparable and of limited range. In a coarsening system, on the other hand, the spatial correlations at time $t$ have a diverging range as $t \rightarrow \infty$, with $\left\langle\sigma_{k}(t) \sigma_{l}(t)\right\rangle \rightarrow 1$, unless specific symmetries of the Hamiltonian are present [12]. This suggests that the large $\Delta t$ limit of the standard four-point correlation $C_{n}\left(\Delta t, t_{\mathrm{w}}\right)$ will be larger in a coarsening system than in glasses, with the growth of spatial two-point correlations obscuring genuine four-point correlation effects.

This is indeed what we will find: to see genuine four-point correlations, we need to consider the "connected" four-point correlation

$$
\begin{aligned}
\mathcal{C}_{l-k}\left(\Delta t, t_{\mathrm{w}}\right)= & \left\langle\sigma_{k}(t) \sigma_{k}\left(t_{\mathrm{w}}\right) \sigma_{l}(t) \sigma_{l}\left(t_{\mathrm{w}}\right)\right\rangle-\left\langle\sigma_{k}(t) \sigma_{k}\left(t_{\mathrm{w}}\right)\right\rangle\left\langle\sigma_{l}(t) \sigma_{l}\left(t_{\mathrm{w}}\right)\right\rangle \\
& -\left\langle\sigma_{k}(t) \sigma_{l}(t)\right\rangle\left\langle\sigma_{k}\left(t_{\mathrm{w}}\right) \sigma_{l}\left(t_{\mathrm{w}}\right)\right\rangle+\left\langle\sigma_{k}(t) \sigma_{l}\left(t_{\mathrm{w}}\right)\right\rangle\left\langle\sigma_{k}\left(t_{\mathrm{w}}\right) \sigma_{l}(t)\right\rangle,
\end{aligned}
$$

which differs from the standard version by the terms in the second line. One observes that the first of these just cancels the residual term from the four-point average in the limit $\Delta t \rightarrow \infty$. We therefore expect that $\mathcal{C}_{n}\left(\Delta t, t_{\mathrm{w}}\right) \rightarrow 0$ for large $\Delta t$ : the connected definition eliminates the uninteresting contributions from spatial two-point correlations. The second term in the second line of (2) can then be motivated as compensating for the first one at short times $\Delta t$, ensuring that, like $C_{n}, \mathcal{C}_{n}$ vanishes at $\Delta t=0$. We note finally that $\mathcal{C}_{n}$ is even in $n$ as was the case for $C_{n}$; at $n=0$, the definition (2) in fact implies that $\mathcal{C}_{0}=0$ at all times.

We will also consider the four-point susceptibilities associated with (1), (2). These are defined as

$$
\chi\left(\Delta t, t_{\mathrm{w}}\right)=\sum_{n=-\infty}^{\infty} C_{n}\left(\Delta t, t_{\mathrm{w}}\right) \quad \text { and } \quad \mathcal{X}\left(\Delta t, t_{\mathrm{w}}\right)=\sum_{n=-\infty}^{\infty} \mathcal{C}_{n}\left(\Delta t, t_{\mathrm{w}}\right) .
$$

The layout of this paper is as follows. First we derive an exact expression for the connected four-point correlation $\mathcal{C}_{n}\left(\Delta t, t_{\mathrm{w}}\right)$ in Section 1, In Section 2 the equilibrium behaviour of the standard and connected four-point functions is discussed. Scalings of 
the connected four-point correlation and its associated susceptibility for non-equilibrium coarsening dynamics are analysed in Section 3. We then interpret our results in Section 4 in terms of the random-walk dynamics of domain walls. The behaviour of the standard four-point functions is briefly presented in Section 5. We conclude in the final Section.

\section{Derivation of the Connected Four-Point Correlation}

In this section we analyse the dynamics of the Glauber-Ising chain [14, quenched from a random initial configuration to some temperature $T \geq 0$. To recap briefly, the model has Hamiltonian $\mathcal{H}=-\sum_{i} \sigma_{i} \sigma_{i+1}$, where $\sigma_{i}= \pm 1(i=1, \ldots N)$ are $N$ Ising spins subject to periodic boundary conditions. Glauber dynamics consists in each spin $\sigma_{i}$ flipping with rate $\frac{1}{2}\left[1-\frac{1}{2} \gamma \sigma_{i}\left(\sigma_{i-1}+\sigma_{i+1}\right)\right]$, where $\gamma=\tanh (2 / T)$.

General expressions for two-time multispin correlation functions in the GlauberIsing chain are given in [15], for the finite model quenched at $t=0$ from equilibrium at an initial temperature $T_{\mathrm{i}}>0$ to arbitrary $T \geq 0$. Let us now recall some results relevant for the present analysis: in the thermodynamic limit $N \rightarrow \infty$ and for a quench from a random initial state $T_{\mathrm{i}} \rightarrow \infty$ we have the following representations

$$
\begin{aligned}
& \left\langle\sigma_{i}(t) \sigma_{j}\left(t_{\mathrm{w}}\right)\right\rangle=\mathrm{e}^{-\Delta t} I_{i-j}(\gamma \Delta t)+\mathcal{E}_{i, j}^{(j)}, \\
& \left\langle\sigma_{i_{1}}(t) \sigma_{i_{2}}(t) \sigma_{j_{1}}\left(t_{\mathrm{w}}\right) \sigma_{j_{2}}\left(t_{\mathrm{w}}\right)\right\rangle=\left[\mathcal{F}_{i_{1}, i_{2}}^{\left(j_{1}, j_{2}\right)}+H_{i_{2}-i_{1}}(2 \Delta t)\right] H_{j_{2}-j_{1}}\left(2 t_{\mathrm{w}}\right) \\
& -\left[+\mathrm{e}^{-\Delta t} I_{i_{1}-j_{1}}(\gamma \Delta t)+\mathcal{E}_{i_{1}, j_{1}}^{\left(j_{1}, j_{2}\right)}\right]\left[-\mathrm{e}^{-\Delta t} I_{i_{2}-j_{2}}(\gamma \Delta t)+\mathcal{E}_{i_{2}, j_{2}}^{\left(j_{1}, j_{2}\right)}\right] \\
& +\left[-\mathrm{e}^{-\Delta t} I_{i_{1}-j_{2}}(\gamma \Delta t)+\mathcal{E}_{i_{1}, j_{2}}^{\left(j_{1}, j_{2}\right)}\right]\left[+\mathrm{e}^{-\Delta t} I_{i_{2}-j_{1}}(\gamma \Delta t)+\mathcal{E}_{i_{2}, j_{1}}^{\left(j_{1}, j_{2}\right)}\right],
\end{aligned}
$$

for two and four-spin two-time correlation functions. In (5) the indices must satisfy $i_{1}<i_{2}$ and $j_{1}<j_{2}$. The general form of the functions $\mathcal{E}$ and $\mathcal{F}$ is

$$
\begin{aligned}
& \mathcal{E}_{i_{\varepsilon}, j_{\nu}}^{\boldsymbol{j}}=\sum_{p}\left[\prod_{\lambda=1}^{\operatorname{dim}(\boldsymbol{j})} \operatorname{sgn}\left(j_{\lambda}-p\right)\right] \mathrm{e}^{-\Delta t} I_{i_{\varepsilon}-p}(\gamma \Delta t) H_{j_{\nu}-p}\left(2 t_{\mathrm{w}}\right), \\
& \mathcal{F}_{i_{\varepsilon}, i_{\delta}}^{\boldsymbol{j}}=\sum_{p, q}\left[\prod_{\lambda=1}^{\operatorname{dim}(\boldsymbol{j})} \operatorname{sgn}\left(j_{\lambda}-p\right) \operatorname{sgn}\left(j_{\lambda}-q\right)\right] \mathrm{e}^{-2 \Delta t} I_{i_{\varepsilon}-p}(\gamma \Delta t) I_{i_{\delta}-q}(\gamma \Delta t) H_{q-p}\left(2 t_{\mathrm{w}}\right) .
\end{aligned}
$$

In (6) and (7) the products over the sign-functions $\operatorname{sgn}(x)$, satisfying $\operatorname{sgn}(0)=0$ and $\operatorname{sgn}(x)=\frac{x}{|x|}$ otherwise, involve the indices of all spins at the earlier time $t_{\mathrm{w}}$; so when substituting (6) into (44) there is only one factor $\operatorname{sgn}(j-p)$ while in (5) we have two factors $\operatorname{sgn}\left(j_{1}-p\right) \operatorname{sgn}\left(j_{2}-p\right)$. The summations over $p, q$ in (6), (7) are to be taken over the entire chain $-\infty<p, q<\infty$. Finally, the functions $I_{q}(t)$ denote modified Bessel functions [16] while the $H_{q}(t)$ have the representation [15]

$$
H_{q}(t)=\frac{\gamma}{2} \int_{0}^{t} \mathrm{~d} \tau \mathrm{e}^{-\tau}\left[I_{q-1}(\gamma \tau)-I_{q+1}(\gamma \tau)\right] .
$$

The physical meaning of $H$ is

$$
\left\langle\sigma_{i}(t) \sigma_{j}(t)\right\rangle=H_{j-i}(2 t),
$$


but this holds for $i<j$ only: in contrast to the two-spin correlation (9), the function $H_{q}(t)$ is odd in $q$ and zero for $q=0$. Further properties of $H$, which are summarized in [15], are recalled below as and when required.

Let us now focus on the connected two-time correlation $\mathcal{C}_{l-k}\left(\Delta t, t_{\mathrm{w}}\right)$ defined in (2). In order to be able to express the four-spin term using (5) we require $k<l$; below, $n$ always stands for $l-k$ and is assumed to be positive. Also substituting (44) and (9) for the corresponding two-spin correlations gives, after some rearranging,

$$
\begin{aligned}
\mathcal{C}_{n}\left(\Delta t, t_{\mathrm{w}}\right)=[ & \left.\mathcal{F}_{k, l}^{(k, l)}+H_{n}(2 \Delta t)-H_{n}(2 t)\right] H_{n}\left(2 t_{\mathrm{w}}\right) \\
& -\left[\mathcal{E}_{k, k}^{(k)}-\mathcal{E}_{k, k}^{(k, l)}\right]\left[\mathcal{E}_{k, k}^{(k)}+\mathcal{E}_{k, k}^{(k, l)}+2 \mathrm{e}^{-\Delta t} I_{0}(\gamma \Delta t)\right] \\
& +\left[\mathcal{E}_{k, l}^{(l)}+\mathcal{E}_{k, l}^{(k, l)}\right]\left[\mathcal{E}_{k, l}^{(l)}-\mathcal{E}_{k, l}^{(k, l)}+2 \mathrm{e}^{-\Delta t} I_{n}(\gamma \Delta t)\right] .
\end{aligned}
$$

Here we have used $\mathcal{E}_{l, l}^{(l)}=\mathcal{E}_{k, k}^{(k)}, \mathcal{E}_{l, k}^{(k)}=\mathcal{E}_{k, l}^{(l)}, \mathcal{E}_{l, l}^{(k, l)}=-\mathcal{E}_{k, k}^{(k, l)}$ and $\mathcal{E}_{l, k}^{(k, l)}=-\mathcal{E}_{k, l}^{(k, l)}$. These properties follow directly from the definition (6) of $\mathcal{E}$ and reflect symmetries like $\left\langle\sigma_{k}(t) \sigma_{l}\left(t_{\mathrm{w}}\right)\right\rangle=\left\langle\sigma_{l}(t) \sigma_{k}\left(t_{\mathrm{w}}\right)\right\rangle$. The problem of analysing $\mathcal{C}_{n}\left(\Delta t, t_{\mathrm{w}}\right)$ is now reduced to rewriting (10) in a convenient form. To this end one could utilize the closed representations for $\mathcal{E}$ and $\mathcal{F}$ derived in [15]. These were, however, constructed for cases where the spins at the earlier time $t_{\mathrm{w}}$ are at a fixed and small distance. In the current context this distance is given by $n$ and we are interested in studying the scaling behaviour for $n \rightarrow \infty$ or working out the infinite sum over $n$ in (3). It is therefore necessary to develop a new approach for dealing with the expression (10).

As we show in the following it is convenient to rearrange the sums $\mathcal{E}$ and $\mathcal{F}$. Let us first consider the sums $\mathcal{E}$, appearing in (10) only in very particular combinations. After substitution of (6) and a shift in the summation variable we obtain, for instance,

$$
\mathcal{E}_{k, k}^{(k)} \pm \mathcal{E}_{k, k}^{(k, l)}=\sum_{p}[1 \pm \operatorname{sgn}(n+p)] \operatorname{sgn}(p) \mathrm{e}^{-\Delta t} I_{p}(\gamma \Delta t) H_{p}\left(2 t_{\mathrm{w}}\right) .
$$

This sum obviously reduces to a semi-infinite one due to the factor in the square brackets, for either choice of the sign. In order to lighten the notation and for the subsequent analysis it is convenient to introduce the weight function

$$
w_{q}=\left\{\begin{array}{lll}
1 & & q=0 \\
2 & \text { for } \quad & 1 \leq q<n \\
1 & & q=n
\end{array} .\right.
$$

We also use a slight modification of $H$

$$
\widehat{H}_{q}(t)=\delta_{q, 0}+H_{q}(t),
$$

and define

$$
\begin{aligned}
& \mathcal{S}_{q}=\mathrm{e}^{-\Delta t} I_{0}(\gamma \Delta t) \widehat{H}_{q}\left(2 t_{\mathrm{w}}\right)+2 \sum_{p=1}^{\infty} \mathrm{e}^{-\Delta t} I_{p}(\gamma \Delta t) H_{q+p}\left(2 t_{\mathrm{w}}\right), \\
& \mathcal{T}_{q}=\mathrm{e}^{-\Delta t} I_{n}(\gamma \Delta t) \widehat{H}_{q}\left(2 t_{\mathrm{w}}\right)+2 \sum_{p=1}^{\infty} \mathrm{e}^{-\Delta t} I_{n+p}(\gamma \Delta t) H_{q+p}\left(2 t_{\mathrm{w}}\right) .
\end{aligned}
$$


In terms of (12 15) the two combinations of $\mathcal{E}$ 's in (11) can then easily be shown to equal

$$
\begin{aligned}
& \mathcal{E}_{k, k}^{(k)}-\mathcal{E}_{k, k}^{(k, l)}=\mathcal{T}_{n}, \\
& \mathcal{E}_{k, k}^{(k)}+\mathcal{E}_{k, k}^{(k, l)}+2 \mathrm{e}^{-\Delta t} I_{0}(\gamma \Delta t)=\mathcal{S}_{0}+\sum_{q=0}^{n} w_{q} \mathrm{e}^{-\Delta t} I_{q}(\gamma \Delta t) \widehat{H}_{q}\left(2 t_{\mathrm{w}}\right) .
\end{aligned}
$$

Similarly we find for the other two combinations of $\mathcal{E}$ 's in (10)

$$
\begin{aligned}
& \mathcal{E}_{k, l}^{(l)}+\mathcal{E}_{k, l}^{(k, l)}=\mathcal{S}_{n}, \\
& \mathcal{E}_{k, l}^{(l)}-\mathcal{E}_{k, l}^{(k, l)}+2 \mathrm{e}^{-\Delta t} I_{n}(\gamma \Delta t)=\mathcal{T}_{0}+\sum_{q=0}^{n} w_{q} \mathrm{e}^{-\Delta t} I_{n-q}(\gamma \Delta t) \widehat{H}_{q}\left(2 t_{\mathrm{w}}\right) .
\end{aligned}
$$

Next consider the double sum $\mathcal{F}$. Here the relevant combination is $\mathcal{F}_{k, l}^{(k, l)}+H_{n}(2 \Delta t)-$ $H_{n}(2 t)$. Based on the identity $I_{q}(x+y)=\sum_{p} I_{p}(x) I_{q+p}(y)$ and (8) one verifies that

$$
H_{l-k}(2 t)-H_{l-k}(2 \Delta t)=\sum_{p, q} \mathrm{e}^{-2 \Delta t} I_{k-p}(\gamma \Delta t) I_{l-q}(\gamma \Delta t) H_{q-p}\left(2 t_{\mathrm{w}}\right) .
$$

Expressing $\mathcal{F}$ via (7) and using (20) then yields

$$
\begin{gathered}
\mathcal{F}_{k, l}^{(k, l)}+H_{n}(2 \Delta t)-H_{n}(2 t)=-\sum_{p, q}[1-\operatorname{sgn}(k-p) \operatorname{sgn}(k-q) \operatorname{sgn}(l-p) \operatorname{sgn}(l-q)] \\
\times \mathrm{e}^{-2 \Delta t} I_{k-p}(\gamma \Delta t) I_{l-q}(\gamma \Delta t) H_{q-p}\left(2 t_{\mathrm{w}}\right) .
\end{gathered}
$$

In analogy with (111) the factor in the square bracket in (21) is non-zero only in a restricted range of the summation variables $p, q$. In fact, the two-dimensional infinite sum in (21) may be rewritten as a finite number of one-dimensional semi-infinite sums. From this procedure, which is slightly cumbersome but trivial, and using the notation (12] - 15]) we obtain

$\mathcal{F}_{k, l}^{(k, l)}+H_{n}(2 \Delta t)-H_{n}(2 t)=\sum_{q=0}^{n} w_{q} \mathrm{e}^{-\Delta t} I_{q}(\gamma \Delta t) \mathcal{T}_{q}-\sum_{q=0}^{n} w_{q} \mathrm{e}^{-\Delta t} I_{n-q}(\gamma \Delta t) \mathcal{S}_{q}$.

In terms of equations (16, 19) and (22) our representation (10) for the connected fourpoint correlation $\mathcal{C}_{n}\left(\Delta t, t_{\mathrm{w}}\right)$ is thus transformed into

$$
\begin{aligned}
\mathcal{C}_{n}\left(\Delta t, t_{\mathrm{w}}\right)= & \mathcal{S}_{n} \mathcal{T}_{0}-\mathcal{T}_{n} \mathcal{S}_{0} \\
& +\sum_{q=0}^{n} w_{q} \mathrm{e}^{-\Delta t} I_{q}(\gamma \Delta t)\left[\mathcal{T}_{q} H_{n}\left(2 t_{\mathrm{w}}\right)-\widehat{H}_{q}\left(2 t_{\mathrm{w}}\right) \mathcal{T}_{n}\right] \\
& -\sum_{q=0}^{n} w_{q} \mathrm{e}^{-\Delta t} I_{n-q}(\gamma \Delta t)\left[\mathcal{S}_{q} H_{n}\left(2 t_{\mathrm{w}}\right)-\widehat{H}_{q}\left(2 t_{\mathrm{w}}\right) \mathcal{S}_{n}\right] .
\end{aligned}
$$

Equation (23) forms the basis for our subsequent analysis of $\mathcal{C}_{n}\left(\Delta t, t_{\mathrm{w}}\right)$. Note that up to this point we have not carried out any summations, except for (20). The derivation of (23) relies only on direct cancellations occurring in (10). 


\section{Equilibrium}

A striking feature of (23) is that it allows us to study the equilibrium behaviour of $\mathcal{C}_{n}\left(\Delta t, t_{\mathrm{w}}\right)$, still without working out any sums at all. To see this we first notice that in equilibrium we have, as can be shown [15] from (8) and (13),

$$
\widehat{H}_{q}^{\text {eq }}=\lim _{t_{\mathrm{w}} \rightarrow \infty} \widehat{H}_{q}\left(2 t_{\mathrm{w}}\right)=\xi^{q} \quad \text { for } \quad q \geq 0,
$$

where

$$
\xi=\frac{1-\sqrt{1-\gamma^{2}}}{\gamma}=\tanh (1 / T)
$$

Due to the exponential dependence of $\widehat{H}_{q}^{\text {eq }}$ on $q$, the expressions (14), (15) for $\mathcal{S}, \mathcal{T}$ satisfy in equilibrium

$$
\mathcal{S}_{q}^{\mathrm{eq}}=\xi^{q} \mathcal{S}_{0}^{\mathrm{eq}} \quad \text { and } \quad \mathcal{T}_{q}^{\mathrm{eq}}=\xi^{q} \mathcal{T}_{0}^{\mathrm{eq}}
$$

It therefore follows immediately from (23) that our connected four-point correlation vanishes in equilibrium, i.e.,

$$
\mathcal{C}_{n}^{\mathrm{eq}}(\Delta t)=\lim _{t_{\mathrm{w}} \rightarrow \infty} \mathcal{C}_{n}\left(\Delta t, t_{\mathrm{w}}\right)=0
$$

for all $n, \Delta t \geq 0$ and at any temperature $T>0$ as specified via $\gamma$. Looking back at the definition of $\mathcal{C}_{n}\left(\Delta t, t_{\mathrm{w}}\right)$ in equation (2), this implies an exact decomposition of four-spin two-time correlations into pairwise correlations. In other words, in equilibrium there are no genuine four-point correlations.

Based on the general expressions given in [15] we have verified that four-point correlations $\left\langle\sigma_{k}(t) \sigma_{l_{1}}\left(t_{\mathrm{w}}\right) \sigma_{l_{2}}\left(t_{\mathrm{w}}\right) \sigma_{l_{3}}\left(t_{\mathrm{w}}\right)\right\rangle$ likewise factorize. There should be a generic connection between this property and the fact that the Glauber-Ising model maps to free fermions [17]. However, as we will see below, the factorization only holds in equilibrium.

Now consider for comparison the standard four-point correlation function (11). It can be expressed in terms of the connected one, Equation (2), via

$$
\begin{aligned}
C_{l-k}\left(\Delta t, t_{\mathrm{w}}\right)= & \mathcal{C}_{l-k}\left(\Delta t, t_{\mathrm{w}}\right) \\
& +\left\langle\sigma_{k}(t) \sigma_{l}(t)\right\rangle\left\langle\sigma_{k}\left(t_{\mathrm{w}}\right) \sigma_{l}\left(t_{\mathrm{w}}\right)\right\rangle-\left\langle\sigma_{k}(t) \sigma_{l}\left(t_{\mathrm{w}}\right)\right\rangle\left\langle\sigma_{k}\left(t_{\mathrm{w}}\right) \sigma_{l}(t)\right\rangle .
\end{aligned}
$$

The first term vanishes in equilibrium, and so $C_{n}^{\mathrm{eq}}(\Delta t)=C_{n}\left(\Delta t, t_{\mathrm{w}} \rightarrow \infty\right)$ can be expressed purely in terms of two-point correlation functions. This suggests that the standard four-point function $C_{l-k}\left(\Delta t, t_{\mathrm{w}}\right)$ is strongly biased by pairwise correlations and thus not suitable for revealing genuine four-point correlation effects.

\section{Non-Equilibrium}

The significance of measuring connected four-point correlations becomes even clearer when considering non-equilibrium coarsening dynamics. For the sake of simplicity we focus on a zero-temperature quench, i.e., $\gamma=1$. The scaling of $\mathcal{C}_{n}\left(\Delta t, t_{\mathrm{w}}\right)$ in the limit of large times $\Delta t, t_{\mathrm{w}} \rightarrow \infty$ and distances $n \rightarrow \infty$ is then expected to be of the form

$$
\mathcal{C}_{n}\left(\Delta t, t_{\mathrm{w}}\right) \sim f_{C}\left(\frac{\Delta t}{2 t_{\mathrm{w}}}, \frac{|n|}{2 \sqrt{t_{\mathrm{w}}}}\right) .
$$


Formally, the long-time and long-distance limit is taken at fixed values of the scaling variables

$$
\alpha=\frac{\Delta t}{2 t_{\mathrm{w}}} \quad \text { and } \quad \eta=\frac{|n|}{2 \sqrt{t_{\mathrm{w}}}} .
$$

The first of these, $\alpha$, measures the observation time interval $\Delta t$ in units of the system's age $t_{\mathrm{w}}$ while $\eta$ is the ratio of distances $n$ over the typical domain size, bearing in mind that the latter scales as $O\left(\sqrt{t_{\mathrm{w}}}\right)$. The factors of $\frac{1}{2}$ in the definitions of $\alpha, \eta$ are included for mathematical convenience in what follows.

In order to obtain the scaling function $f_{C}(\alpha, \eta)$ a leading order asymptotic expansion of (23) is required. To this end we use the asymptotic formula [16]

$$
\mathrm{e}^{-t} I_{q}(t) \sim \frac{1}{\sqrt{2 \pi t}} \mathrm{e}^{-q^{2} /(2 t)}
$$

which applies for $q, t \rightarrow \infty$ with $q^{2} / t$ fixed. In the same limit we have, by combining (8) with (31) and the identity $I_{q-1}(t)-I_{q+1}(t)=\frac{2 q}{t} I_{q}(t)$, and setting $\gamma=1$,

$$
H_{q}(t) \sim \Phi\left(\frac{q}{\sqrt{2 t}}\right) \text { with } \Phi(x)=\frac{2}{\sqrt{\pi}} \int_{x}^{\infty} \mathrm{d} u \mathrm{e}^{-u^{2}} .
$$

The function $\Phi(x)$ is in fact just the complementary error function $\Phi(x)=\operatorname{erfc}(x)=$ $1-\operatorname{erf}(x)$; we use the symbol $\Phi$ to keep the notation compact. When substituting (31), (32) into (14), (15) the sums defining $\mathcal{S}_{q}, \mathcal{T}_{q}$ turn into Riemann-sums such that

$$
\begin{aligned}
& \mathcal{S}_{q} \sim \frac{2}{\sqrt{\pi}} \int_{0}^{\infty} \mathrm{d} x \mathrm{e}^{-x^{2}} \Phi\left(\frac{q}{2 \sqrt{t_{\mathrm{w}}}}+\sqrt{\frac{\Delta t}{2 t_{\mathrm{w}}}} x\right), \\
& \mathcal{T}_{q} \sim \frac{2}{\sqrt{\pi}} \int_{0}^{\infty} \mathrm{d} x \mathrm{e}^{-(x+n / \sqrt{2 \Delta t})^{2}} \Phi\left(\frac{q}{2 \sqrt{t_{\mathrm{w}}}}+\sqrt{\frac{\Delta t}{2 t_{\mathrm{w}}}} x\right) .
\end{aligned}
$$

Using the expansions (31)-(34) in (23) and taking the scaling limit also turns the sums explicitly appearing in (23) into integrals. In terms of our scaling variables $\alpha, \eta$ the scaling function $f_{C}(\alpha, \eta)$ is thus

$$
\begin{aligned}
f_{C}(\alpha, \eta)=\frac{4}{\alpha \pi} & \left\{\int_{0}^{\infty} \mathrm{d} u \int_{0}^{\infty} \mathrm{d} v \mathrm{e}^{-\frac{1}{\alpha}\left[u^{2}+(\eta+v)^{2}\right]}[\Phi(\eta+u) \Phi(v)-\Phi(u) \Phi(\eta+v)]\right. \\
& +\int_{0}^{\eta} \mathrm{d} u \int_{0}^{\infty} \mathrm{d} v \mathrm{e}^{-\frac{1}{\alpha}\left[u^{2}+(\eta+v)^{2}\right]}[\Phi(u+v) \Phi(\eta)-\Phi(u) \Phi(\eta+v)] \\
& \left.-\int_{0}^{\eta} \mathrm{d} u \int_{0}^{\infty} \mathrm{d} v \mathrm{e}^{-\frac{1}{\alpha}\left[(\eta-u)^{2}+v^{2}\right]}[\Phi(u+v) \Phi(\eta)-\Phi(u) \Phi(\eta+v)]\right\} .
\end{aligned}
$$

Equation (35) is suitable for numerical evaluation; the resulting plots of $f_{C}(\alpha, \eta)$ are shown inFig. 1. One sees clearly that there are non-trivial four-point correlations in the non-equilibrium coarsening dynamics of the Glauber-Ising chain. We notice also that $f_{C}(\alpha, \eta)$ is negative throughout (the plot shows the modulus); we will find an explanation for this feature in Section 4 below. Because of the rather rich structure in $f_{C}(\alpha, \eta)$ let us next discuss its various scaling regimes.

First consider $\alpha \gg 1$, which corresponds to $\Delta t \gg t_{\mathrm{w}}$. The behaviour of $f_{C}(\alpha, \eta)$ in this regime is easily obtained form (35) by Taylor expanding the exponentials in $1 / \alpha$. 


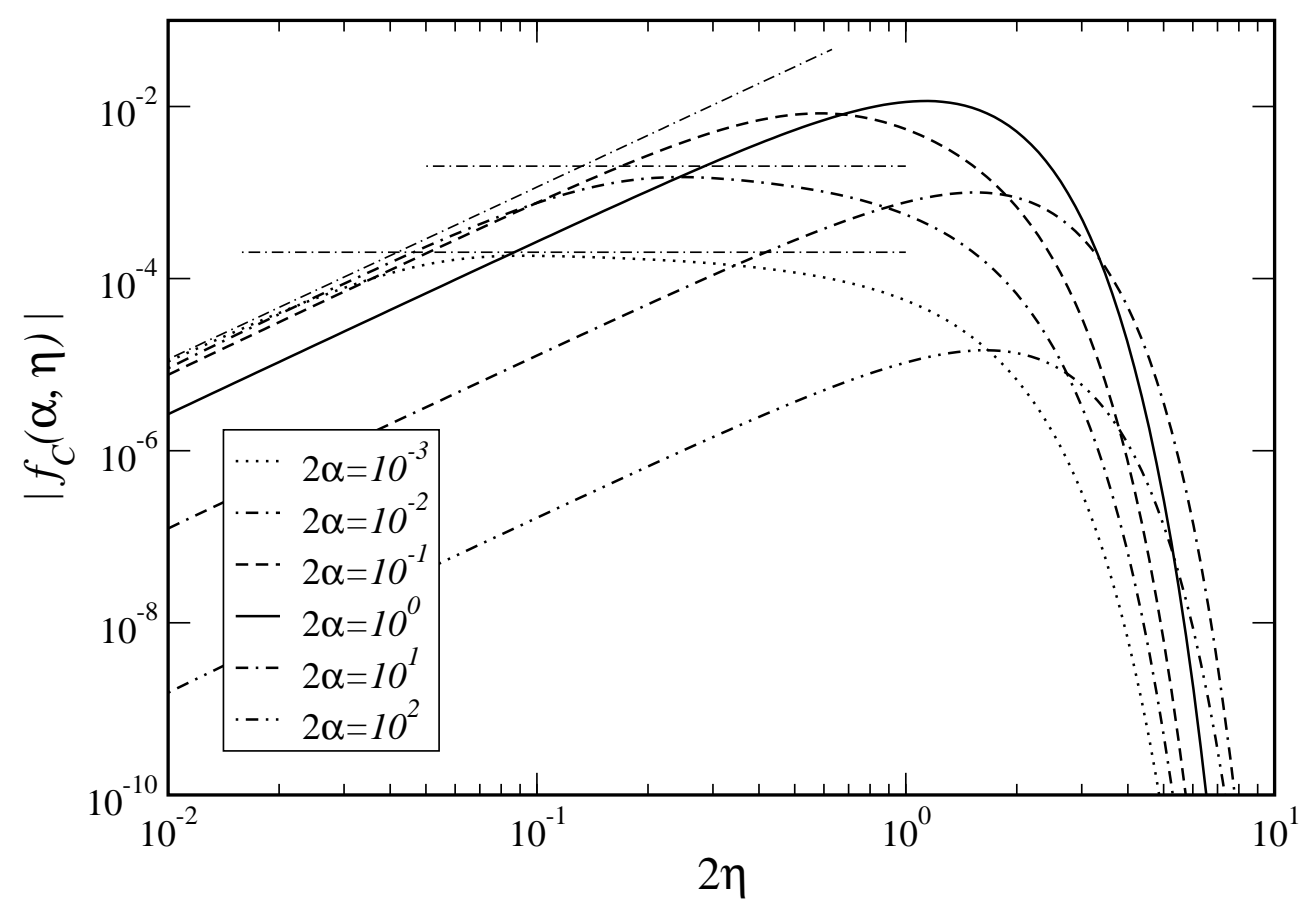

Figure 1. Modulus of the scaling function $f_{C}(\alpha, \eta)$ of the connected four-point correlation $\mathcal{C}_{n}\left(\Delta t, t_{\mathrm{w}}\right)$ versus distance $2 \eta=|n| / \sqrt{t_{\mathrm{w}}}$ for various time ratios $2 \alpha=\Delta t / t_{\mathrm{w}}$. The curves are obtained by numerical evaluation of the exact scaling functions (35). The dashed-dotted straight lines are the asymptotes (42) and (43), where the sloping line corresponds to (42) for the regime $\eta^{2} \ll \alpha \ll 1$ while the horizontal ones represent (433) with $2 \alpha=10^{-3}, 10^{-2}$ and apply in the regime $\alpha \ll \eta^{2} \ll 1$. The data is discussed below and interpreted in Section 4

The zeroth order contributions, where the exponentials are replaced by unity, vanish: in the first integral in (35) the combination of $\Phi$ 's is antisymmetric under exchanging $u, v$ while the second and third integral cancel. So the leading behaviour of $f_{C}(\alpha, \eta)$ follows from first-order contributions where, for the same reasons, various terms drop out. The remaining integrals, which are of the type $\int \mathrm{d} x x^{i} \Phi(x)$ with $i=0,1,2$ and $\int \mathrm{d} x \int \mathrm{d} y(x+y) \Phi(x+y)$, are solvable [16] and lead to the large $\alpha$ expansion

$$
\begin{gathered}
f_{C}(\alpha, \eta)=-\frac{2}{3 \pi^{2}}\left\{12 \eta^{2} \mathrm{e}^{-\eta^{2}}-2\left[\left(1+6 \eta^{2}\right)-\left(1+\eta^{2}\right) \mathrm{e}^{-\eta^{2}}\right] \sqrt{\pi} \eta \Phi(\eta)\right. \\
\left.-\left(3+2 \eta^{2}\right) \pi \eta^{2} \Phi^{2}(\eta)\right\} \frac{1}{\alpha^{2}}+O\left(\frac{1}{\alpha^{3}}\right) .
\end{gathered}
$$

This means that the magnitude of four-point correlations drops as $1 / \alpha^{2}$ for $\alpha \gg 1$, at all distances $\eta$. The plots of the exact $f_{C}(\alpha, \eta)$ in Fig. 团 for $2 \alpha=10^{1}, 10^{2}$ illustrate this nicely. Details of the shape of $f_{C}(\alpha, \eta)$ at large $\alpha$ are revealed by expanding (36) for $\eta \ll 1$ and $\eta \gg 1$, i.e., distances $n$ far below and far above the typical domain-size at time $t_{\mathrm{w}}$. In the former case we simply Taylor expand in $\eta$ while in the latter we use 16] that $\Phi(\eta)=1 /(\sqrt{\pi} \eta) \mathrm{e}^{-\eta^{2}}\left[1-1 /\left(2 \eta^{2}\right)+O\left(\eta^{-4}\right)\right]$. The leading terms are

$$
f_{C}(\alpha, \eta) \sim-\frac{2}{\pi}\left(\frac{4}{\pi}-1\right) \frac{\eta^{2}}{\alpha^{2}} \quad \text { for } \quad \eta^{2} \ll 1 \ll \alpha,
$$




$$
f_{C}(\alpha, \eta) \sim-\frac{8}{3 \pi^{2} \alpha^{2}} \mathrm{e}^{-\eta^{2}} \quad \text { for } \quad 1 \ll \alpha, \eta^{2} .
$$

Again the plots in Fig. 1 for $2 \alpha=10^{1}, 10^{2}$ clearly show the power-law behaviour given by (37) and the Gaussian cutoff (38).

Now we turn to $\alpha \ll 1$ or $\Delta t \ll t_{\mathrm{w}}$. To obtain expansions of $f_{C}(\alpha, \eta)$ in this regime it is necessary to rearrange (B5). It is further convenient to introduce

$$
\hat{f}_{C}(\alpha, \rho)=f_{C}(\alpha, \sqrt{\alpha} \rho) \quad \text { with } \quad \rho=\frac{\eta}{\sqrt{\alpha}}=\frac{n}{\sqrt{2 \Delta t}} .
$$

The scaling variable $\rho$ may be viewed as an alternative measure for distance and thus replaces $\eta$. In this notation and by shifting and scaling the integration variables equation (35) can be re-expressed as

$$
\begin{aligned}
\hat{f}_{C}(\alpha, \rho)=\frac{4}{\pi} & \left\{\int_{0}^{\infty} \mathrm{D} u \int_{\rho}^{\infty} \mathrm{D} v\left[\Phi_{\alpha}(\rho+u) \Phi_{\alpha}(v-\rho)-\Phi_{\alpha}(u) \Phi_{\alpha}(v)\right]\right. \\
& +\int_{0}^{\rho} \mathrm{D} u \int_{\rho}^{\infty} \mathrm{D} v\left[\Phi_{\alpha}(v+u-\rho) \Phi_{\alpha}(\rho)-\Phi_{\alpha}(u) \Phi_{\alpha}(v)\right] \\
& \left.-\int_{0}^{\rho} \mathrm{D} u \int_{0}^{\infty} \mathrm{D} v\left[\Phi_{\alpha}(v-u+\rho) \Phi_{\alpha}(\rho)-\Phi_{\alpha}(\rho-u) \Phi_{\alpha}(\rho+v)\right]\right\} .
\end{aligned}
$$

Here we have introduced the short-hands $\mathrm{D} x=\mathrm{e}^{-x^{2}} \mathrm{~d} x$ and $\Phi_{\alpha}(x)=\Phi(\sqrt{\alpha} x)$. Equation (401) is suitable for studying the small $\alpha$ regime of $f_{C}(\alpha, \eta)$. Two cases have to be distinguished: we can expand around $\alpha=0$ either at fixed $\rho$ or at fixed $\eta$. When keeping $\rho$ fixed we effectively look at distances $\eta=\rho \sqrt{\alpha}=O(\sqrt{\alpha})$, while obviously $\eta=O(1)$ if we fix $\eta$. Because the $\rho$ and $\eta$ length scales become disparate for $\alpha \rightarrow 0$, separate expansions must be made.

The shape of $f_{C}(\alpha, \eta)$ for small $\alpha$ and fixed $\rho$ immediately follows from (40) by Taylor expanding the functions $\Phi_{\alpha}(x)=\Phi(\sqrt{\alpha} x)$ in $\sqrt{\alpha}$. This turns the integrands in (40) into Gaussians (contained in $\mathrm{D} u, \mathrm{D} v$ ) with polynomial factors. Evaluating the integrals gives

$$
\hat{f}_{C}(\alpha, \rho)=-\frac{4}{\pi^{2}}\left\{1-\left[\mathrm{e}^{-\rho^{2}}+\sqrt{\pi} \rho(2-\Phi(\rho))\right]\left[\mathrm{e}^{-\rho^{2}}-\sqrt{\pi} \rho \Phi(\rho)\right]\right\} \alpha+O\left(\alpha^{3 / 2}\right) .
$$

To understand the result (41) it is instructive to consider the limits $\rho \ll 1$ and $\rho \gg 1$, corresponding to distances $n \ll \sqrt{\Delta t}$ and $n \gg \sqrt{\Delta t}$, respectively. One finds to leading order and in terms of $\alpha$ and $\eta$

$$
\begin{array}{ll}
f_{C}(\alpha, \eta) \sim-\frac{4}{\pi}\left(1-\frac{2}{\pi}\right) \eta^{2} \quad \text { for } \quad \eta^{2} \ll \alpha \ll 1, \\
f_{C}(\alpha, \eta) \sim-\frac{4}{\pi^{2}} \alpha \quad & \text { for } \quad \alpha \ll \eta^{2} \ll 1 .
\end{array}
$$

We note that at small $\rho$ the leading term in (41) is $\rho^{2} \alpha=\eta^{2}$ and thus (42) follows. So $f_{C}(\alpha, \eta)$ initially grows as $\eta^{2}$ independently of $\alpha$. But for $\eta \approx \sqrt{\alpha}$ four-point correlations level off at a plateau of height $O(\alpha)$, as given by equation (43). The asymptotes (42), (43) are shown in Fig. 囵,

It remains to discuss the behaviour of $f_{C}(\alpha, \eta)$ for small $\alpha$ and fixed $\eta$. In this case $\rho=\eta / \sqrt{\alpha}$ in (40) diverges for $\alpha \rightarrow 0$. Because of the Gaussian weights in $\mathrm{D} u, \mathrm{D} v$ 
only integrals containing the neighborhood of $u=v=0$ then contribute significantly to $f_{C}(\alpha, \eta)$. This holds for the third integral in (40), but not for the other two. The first one, for instance, satisfies the bound

$$
\left|\frac{4}{\pi} \int_{0}^{\infty} \mathrm{D} u \int_{\rho}^{\infty} \mathrm{D} v\left[\Phi_{\alpha}(\rho+u) \Phi_{\alpha}(v-\rho)-\Phi_{\alpha}(u) \Phi_{\alpha}(v)\right]\right| \leq 2 \Phi(\eta) \Phi(\rho) .
$$

This follows from the triangular inequality, the identities $\Phi(\rho)=(2 / \sqrt{\pi}) \int_{\rho}^{\infty} \mathrm{D} x$ and $\Phi(\eta)=\Phi_{\alpha}(\rho)$ and the fact that $\Phi(x)$ is monotonously decreasing. The same bound can be used for the second integral in (40). Extending the $u$-integration range in the third integral to $\int_{0}^{\infty} \mathrm{D} u$ likewise only produces excess contributions of the same size. Therefore, to order $O(\Phi(\eta) \Phi(\rho))$ equation (40) reduces to

$f_{C}(\alpha, \eta) \simeq-\frac{4}{\pi} \int_{0}^{\infty} \mathrm{D} u \int_{0}^{\infty} \mathrm{D} v[\Phi(\sqrt{\alpha}(v-u)+\eta) \Phi(\eta)-\Phi(\eta-\sqrt{\alpha} u) \Phi(\eta+\sqrt{\alpha} v)]$

at small $\alpha$ and fixed $\eta$. Here we have substituted $\Phi_{\alpha}(x)=\Phi(\sqrt{\alpha} x)$ and $\sqrt{\alpha} \rho=\eta$. As will become clear in a moment the above expression has power-law scaling at small $\alpha$. On the other hand, $\Phi(\rho) \sim \sqrt{\alpha} /(\sqrt{\pi} \eta) \mathrm{e}^{-\eta^{2} / \alpha}$ vanishes faster than any power-law for $\alpha \rightarrow 0$. Therefore we may safely ignore the $O(\Phi(\eta) \Phi(\rho))$ contributions discarded above. By Taylor expanding the last representation for $f_{C}(\alpha, \eta)$ in $\sqrt{\alpha}$, which leads to simple Gaussian integrals, we thus finally obtain the small $\alpha$ scaling at fixed $\eta$,

$$
f_{C}(\alpha, \eta)=-\frac{4}{\pi^{2}} \mathrm{e}^{-\eta^{2}}\left[\mathrm{e}^{-\eta^{2}}-\sqrt{\pi} \eta \Phi(\eta)\right] \alpha+O\left(\alpha^{3 / 2}\right) .
$$

Equation (44) tells us that, on the length scale set by $\eta$, four-point correlations decrease linearly with $\alpha$ as $\alpha \rightarrow 0$, for any value of $\eta$. The plots for $2 \alpha=10^{-2}, 10^{-3}$ in Fig. 1 illustrate this. At small and large $\eta=|n| /\left(2 \sqrt{t_{\mathrm{w}}}\right)$ the behaviour of (44) is to leading order

$$
\begin{aligned}
& f_{C}(\alpha, \eta) \sim-\frac{4}{\pi^{2}} \alpha \quad \text { for } \quad \alpha \ll \eta^{2} \ll 1, \\
& f_{C}(\alpha, \eta) \sim-\frac{2 \alpha}{\pi^{2} \eta^{2}} \mathrm{e}^{-2 \eta^{2}} \quad \text { for } \quad \alpha \ll 1 \ll \eta^{2} .
\end{aligned}
$$

The plateau (45) obtained at small $\eta$ matches the one at large $\rho$, Equation (43), as it should. For small $\alpha$ and large $\eta$, finally, $f_{C}(\alpha, \eta)$ has an essentially Gaussian cutoff (46)). The latter occurs at slightly smaller $\eta$ than in the large $\alpha$ case, see (38) and Fig. 1 .

Our discussion of $f_{C}(\alpha, \eta)$ has given us a complete understanding of the spatiotemporal scaling of the connected four-point correlation $\mathcal{C}_{n}\left(\Delta t, t_{\mathrm{w}}\right)$. The various scaling regimes in Fig. 1 are characterized by (37), (38), (42), (43), (45) and (46). We now conclude our analysis of the connected four-point correlation in nonequilibrium coarsening by studying the scaling of the associated four-point susceptibility. From its definition (3), the properties $\mathcal{C}_{-n}\left(\Delta t, t_{\mathrm{w}}\right)=\mathcal{C}_{n}\left(\Delta t, t_{\mathrm{w}}\right), \mathcal{C}_{0}\left(\Delta t, t_{\mathrm{w}}\right)=0$ and the scaling (29) one finds in the large-time limit $\Delta t, t_{\mathrm{w}} \rightarrow \infty$ at fixed $\alpha$,

$$
\mathcal{X}\left(\Delta t, t_{\mathrm{w}}\right)=2 \sum_{n=1}^{\infty} \mathcal{C}_{n}\left(\Delta t, t_{\mathrm{w}}\right) \sim 2 \sum_{n=1}^{\infty} f_{C}\left(\frac{\Delta t}{2 t_{\mathrm{w}}}, \frac{n}{2 \sqrt{t_{\mathrm{w}}}}\right) \sim 4 \sqrt{t_{\mathrm{w}}} \int_{0}^{\infty} \mathrm{d} \eta f_{C}(\alpha, \eta) .
$$


This defines the scaling function $F_{C}(\alpha)$ for the susceptibility,

$$
\mathcal{X}\left(\Delta t, t_{\mathrm{w}}\right) \sim \sqrt{t_{\mathrm{w}}} F_{C}\left(\frac{\Delta t}{2 t_{\mathrm{w}}}\right) \quad \text { with } \quad F_{C}(\alpha)=4 \int_{0}^{\infty} \mathrm{d} \eta f_{C}(\alpha, \eta) .
$$

Numerical integration of (47) using $f_{C}(\alpha, \eta)$ as given in (35) produces the plots in Fig. 2 . Because $f_{C}(\alpha, \eta)<0$ we also find that $F_{C}(\alpha)$ is negative throughout. Furthermore, $F_{C}(\alpha)$ shows power-law behavior at small as well as large $\alpha$ :

$$
\begin{aligned}
& F_{C}(\alpha)=-\frac{8(\sqrt{2}-1)}{\pi^{3 / 2}} \alpha+O\left(\alpha^{3 / 2}\right) \quad \text { for } \quad \alpha \ll 1, \\
& F_{C}(\alpha)=-\frac{2(8 \sqrt{2}-9)}{5 \pi^{3 / 2}} \frac{1}{\alpha^{2}}+O\left(\frac{1}{\alpha^{3}}\right) \quad \text { for } \quad \alpha \gg 1 .
\end{aligned}
$$

The expansion for large $\alpha$ is obtained by substituting the corresponding expansion (36) of $f_{C}(\alpha, \eta)$ in (47). At small $\alpha$ we split $\int_{0}^{\infty} \mathrm{d} \eta=\int_{0}^{\nu \sqrt{\alpha}} \mathrm{d} \eta+\int_{\nu \sqrt{\alpha}}^{\infty} \mathrm{d} \eta$ in (47) with some $\nu>0$. The integrals correspond to the $\rho$ and $\eta$ length scales where the expansions (41) and (44) of $f_{C}(\alpha, \eta)$ apply, respectively. One easily shows that the contributions to $F_{C}(\alpha)$ from the $\rho$ length scale are $O\left(\alpha^{3 / 2}\right)$ while those from the $\eta$ scale grow as $O(\alpha)$. Therefore the leading term in the small $\alpha$ expansion (48) is given by substituting (44) into (47). The fact that $F_{C}(\alpha)$ vanishes at small and large $\alpha$ is not surprising: as discussed below (2) the connected four-point correlation $\mathcal{C}_{n}\left(\Delta t, t_{\mathrm{w}}\right)$ goes to zero for $\Delta t \rightarrow 0$ as well as $\Delta t \rightarrow \infty$. However, that the approach to these limits is through the power laws (48) and (49) is rather non-trivial. From the arguments above it is clear that the linear scaling at small $\alpha$ is a consequence of the same scaling of the plateau height in $f_{C}(\alpha, \eta)$. We consider next a physical picture which provides some intuition into how this scaling arises.

\section{Random Walk Interpretation}

The exact scaling results summarized in Figure 1 have a lot of structure. To develop a physical understanding, we now use the fact that low-temperature dynamics of the Ising chain can be described in terms of domain walls that perform independent random walks with diffusion rate $\frac{1}{2}$ until they meet, when they annihilate with rate close to unity [18. In equilibrium there is also the reverse process, where pairs of domain walls or "walkers" are created at a small rate.

In a space-time diagram [8], Figure B(a), the spins $\sigma_{k}\left(t_{\mathrm{w}}\right), \sigma_{l}\left(t_{\mathrm{w}}\right), \sigma_{k}(t)$ and $\sigma_{l}(t)$ define the four corners of a rectangle. Spin products are then determined by whether an even or odd number of domain walls cross the relevant edge of the rectangle. For instance $\sigma_{k}\left(t_{\mathrm{w}}\right) \sigma_{k}(t)$ equals 1 if an even number of walkers cross the bottom edge; otherwise it equals -1 . One can then classify all possible situations by the parity of the number of walkers crossing the four edges. Numbering the edges in the order left - right - top - bottom, we will for example denote the situation where an odd number of walkers crosses on the left and bottom as 1001. Because walkers only annihilate or recreate in pairs, the total number of walkers crossing the rectangle has to be even, so that there 


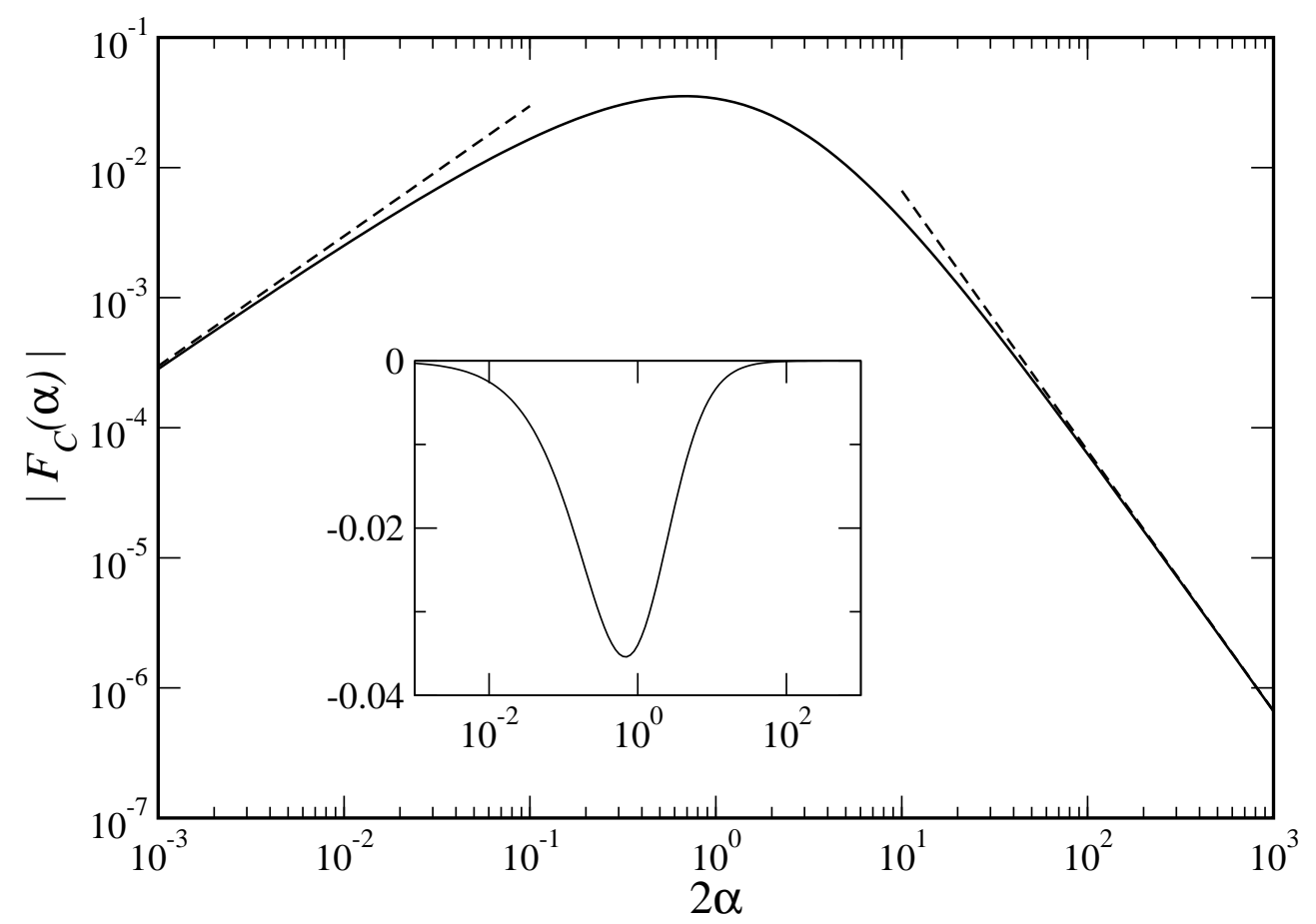

Figure 2. Modulus of the scaling function $F_{C}(\alpha)$ of the four-point susceptibility $\mathcal{X}\left(\Delta t, t_{\mathrm{w}}\right)$ defined in (47), versus $2 \alpha=\Delta t / t_{\mathrm{w}}$ for zero temperature coarsening. Dashed lines represent the asymptotes (48) and (49). Inset: $F_{C}$ on a linear scale.

are eight possible situations. After a short calculation one shows that, in terms of the corresponding probabilities, the connected four-point correlation is

$$
\mathcal{C}_{n}\left(\Delta t, t_{\mathrm{w}}\right)=8\left(p_{0000} p_{1111}+p_{0011} p_{1100}-p_{0101} p_{1010}-p_{0110} p_{1001}\right) .
$$

The last two terms are always identical to each other, due to the spatial mirror symmetry of the problem. The representation (150) is particularly useful when the number of walkers crossing the rectangle is small. We expect this to be the case in the regime $n \ll 1 / c\left(t_{\mathrm{w}}\right)$ and $\sqrt{\Delta t} \ll 1 / c\left(t_{\mathrm{w}}\right)$, where $c\left(t_{\mathrm{w}}\right)$ is the concentration of domain walls at time $t_{\mathrm{w}}$. In the coarsening regime, where $c\left(t_{\mathrm{w}}\right) \sim 1 /\left(2 \sqrt{\pi t_{\mathrm{w}}}\right)$, these conditions translate to $n^{2} \ll t_{\mathrm{w}}$ and $\Delta t \ll t_{\mathrm{w}}$. In equilibrium, on the other hand, $c$ is a constant fixed by the temperature. To include both cases, we simply write $c$ below, even for the coarsening case.

Our restrictions so far still leave open the relative magnitude of $\Delta t$ and $n^{2}$; let us focus first on the case where $\Delta t \ll n^{2}$. The space-time rectangle is then wide in the space-direction and narrow in the time-direction. From this one can deduce the leading contributions to the various probabilities, which are shown in Figure 3(b). We have denoted by $\Gamma$ the probability that a walker will cross a corner of the rectangle. Since $n \gg \sqrt{\Delta t}$, this probability is dominated by the smallness of $\sqrt{\Delta t}$, while the spatial extent of the box is irrelevant. Thus, $\Gamma$ can be calculated as the probability that a walker will cross from one half-space into the other during the time interval $\Delta t$, which is easily found as $\Gamma=c \sqrt{\Delta t / 2 \pi}$. The other quantity that appears in the probabilities is 
(a)

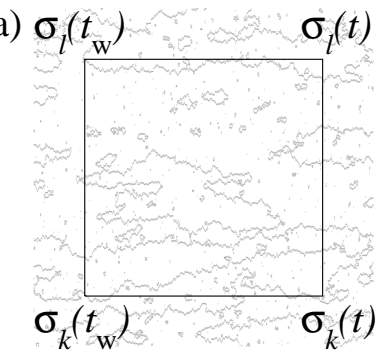

(b)

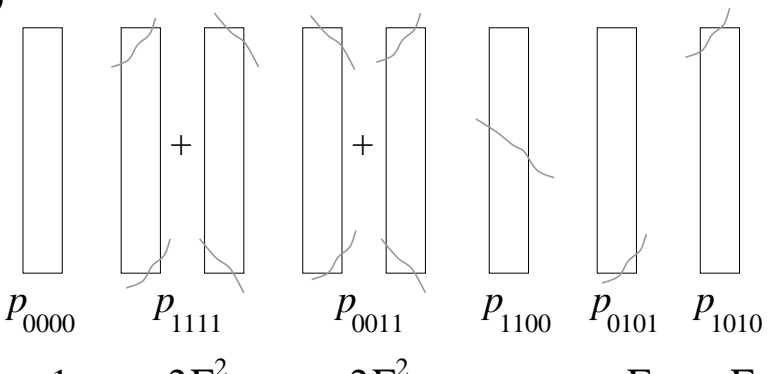

(c)

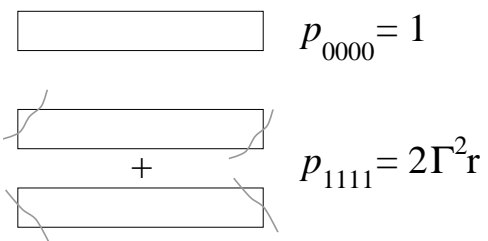

$=2 \Gamma^{2} \mathrm{r}$

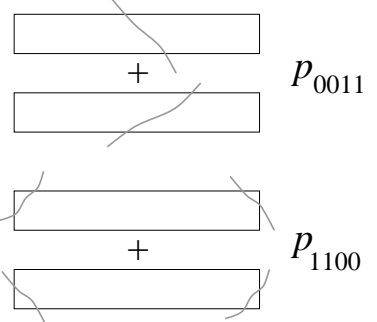

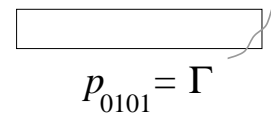

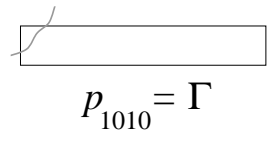

Figure 3. Space-time representation of the connected four-point correlation; grey lines indicate domain-wall trajectories [8]. Panel (a): snapshot of trajectories over a spatial region (vertical) of 500 sites and a time-window (horizontal) of $\Delta t=1500$ for equilibrium dynamics at $T=0.6$. Panel (b), (c): schematic trajectories. See text for discussion.

the factor $r$, which we define to be the joint probability at time $t_{\mathrm{w}}$ of having two walkers at distance $n$, normalized by $c^{2}$. With this normalization, one would have $r=1$ for $n \gg 1 / c$ because correlations between walkers vanish at large distance. At equilibrium, where walkers are uncorrelated at any distance, one in fact has $r=1$ even for $n \ll 1 / c$. In the coarsening situation, on the other hand, $r$ is of order $n c$ for $n \ll 1 / c$ and thus vanishes to leading order. This reflects the effective repulsion between walkers: a walker that has survived the coarsening dynamics up to $t_{\mathrm{w}}$ is not likely to have other walkers within a distance of order $O\left(\sqrt{t_{\mathrm{w}}}\right)=O(1 / c)$. Putting the terms from Figure 3(b) together gives

$\mathcal{C}_{n}\left(\Delta t, t_{\mathrm{w}}\right) \approx 8\left(1 \times 2 \Gamma^{2} r+2 \Gamma^{2} r \times n c-2 \Gamma^{2}\right) \approx 16 \Gamma^{2}(r-1)=\frac{8}{\pi}(r-1) c^{2} \Delta t$,

where we have used the fact that $n c \ll 1$ to neglect the second term. This simple expression explains two important qualitative observations made above. First, in equilibrium, $\mathcal{C}_{n}$ vanishes because $r=1$. Second, in the coarsening case, $\mathcal{C}_{n}$ is negative because $r<1$ when $n \ll 1 / c$. Thus, the sign of $\mathcal{C}_{n}$ arises from the effective repulsion between walkers discussed previously. Quantitatively, using that $r \ll 1$ for $n \ll 1 / c$, the result (51) predicts that

$$
\mathcal{C}_{n} \approx-\frac{8}{\pi} c^{2} \Delta t \sim-\frac{2}{\pi^{2}} \frac{\Delta t}{t_{\mathrm{w}}}=-\frac{4}{\pi^{2}} \alpha .
$$

This is precisely our expansion (43) for the regime $\Delta t \ll n^{2} \ll t_{\mathrm{w}}$, where $\mathcal{C}_{n}$ has an $n$-independent plateau whose height increases linearly with $\Delta t / t_{\mathrm{w}}$. So we now have a 
microscopic picture for the occurrence of this plateau in terms of domain-wall dynamics.

Next we apply similar arguments to the regime where $n$ is small compared to $\sqrt{\Delta t}$, $n^{2} \ll \Delta t \ll t_{\mathrm{w}}$. As shown in Figure 3(c), the space-time rectangle is now extended in the time-direction. As a consequence, the two probabilities $p_{0011}$ and $p_{1100}$ swap their leading contributions. For $p_{0011}$, the leading term is now produced by a single walker crossing the rectangle from top to bottom or from bottom to top. Since $n \ll \sqrt{\Delta t}$, the spatial width of the box can be neglected to leading order, and $p_{0011}$ reduces to the probability of crossing from one halfspace to the other. Bearing in mind that the crossing can occur from the top or the bottom then gives $p_{0011}=2 c \sqrt{\Delta t / 2 \pi}$. For $p_{1100}$, one might naively expect to get a product of two corner-crossing probabilities times a repulsion factor. However, as opposed to the case of $p_{1111}$, the two corner crossings shown can in fact be achieved by a single walker which starts and ends within the interval of size $n$. This gives a leading contribution of $p_{1100}=n c \times n / \sqrt{2 \pi \Delta t}$. The remaining terms are calculated as before, except for the fact that now the corner-crossing probability is $\Gamma=n c / 2$. Assembling all terms, and using again that $r \ll 1$ to neglect $p_{0000} p_{1111}=2 \Gamma^{2} r$, we thus get for the coarsening case

$$
\mathcal{C}_{n}\left(\Delta t, t_{\mathrm{w}}\right) \approx 8\left[2 c \sqrt{\frac{\Delta t}{2 \pi}} \times \frac{n^{2} c}{\sqrt{2 \pi \Delta t}}-2 \Gamma^{2}\right]=-4\left(1-\frac{2}{\pi}\right) n^{2} c^{2} .
$$

This again has the correct negative sign overall. It also predicts that, in this small-n regime, $\mathcal{C}_{n}$ grows quadratically with $n$, with an amplitude independent of $\Delta t$. In fact, using $n^{2} c^{2} \sim n^{2} /\left(4 \pi t_{\mathrm{w}}\right)=\eta^{2} / \pi$, the result (53) coincides with the expansion (42) as it should.

The random walk picture has turned out to be useful for explaining the behaviour of $\mathcal{C}_{n}$ when $n^{2}, \Delta t \ll t_{\mathrm{w}}$. In the remaining regimes discussed in the previous Section, on the other hand, where either $n^{2}$ or $\Delta t$ are large compared to $t_{\mathrm{w}}$, it is less helpful because a large number of annihilating walkers has to be considered. It is then no longer obvious how to estimate the probabilities in (50). Nevertheless, the Gaussian cutoff for

$n^{2} \gg t_{\mathrm{w}}$ that we found in (38), (46) is at least qualitatively reasonable: for length scales $n \gg \sqrt{t_{\mathrm{w}}}$, correlations between random walkers become weak and one should effectively retrieve the equilibrium situation, where $\mathcal{C}_{n}=0$.

\section{Standard Functions out of Equilibrium}

In Section 2 we saw that while the connected four-point correlation $\mathcal{C}_{n}\left(\Delta t, t_{\mathrm{w}}\right)$ and its associated four-point susceptibility $\mathcal{X}\left(\Delta t, t_{\mathrm{w}}\right)$ vanish in equilibrium, the standard functions $C_{n}\left(\Delta t, t_{\mathrm{w}}\right)$ and $\chi\left(\Delta t, t_{\mathrm{w}}\right)$ are biased by two-spin correlations. It is the purpose of this section to show that the same is true for the non-equilibrium coarsening dynamics. The link (28) between the standard and connected four-point correlations allows us to express their difference $\Delta C_{n}\left(\Delta t, t_{\mathrm{w}}\right)=C_{n}\left(\Delta t, t_{\mathrm{w}}\right)-\mathcal{C}_{n}\left(\Delta t, t_{\mathrm{w}}\right)$ purely in terms of two-spin correlations. This makes the analysis of $\Delta C_{n}\left(\Delta t, t_{\mathrm{w}}\right)$ rather simple: spatial correlations are given in (9) in terms of $H_{n}$ while temporal correlations for zero 
temperature coarsening have the exact representation [15]

$$
\left\langle\sigma_{k}(t) \sigma_{l}\left(t_{\mathrm{w}}\right)\right\rangle=\mathrm{e}^{-\left(t+t_{\mathrm{w}}\right)}\left\{I_{n}\left(t+t_{\mathrm{w}}\right)+\int_{0}^{2 t_{\mathrm{w}}} \mathrm{d} \tau I_{n}\left(t+t_{\mathrm{w}}-\tau\right)\left[I_{0}(\tau)+I_{1}(\tau)\right]\right\} .
$$

In the scaling limit $\Delta t, t_{\mathrm{w}}, n \rightarrow \infty$ with $\alpha=\Delta t /\left(2 t_{\mathrm{w}}\right)$ and $\eta=|n| /\left(2 \sqrt{t_{\mathrm{w}}}\right)$ fixed we substitute the expansion (31) into (154). Combining terms according to (28) and some rearranging then produces $\Delta C_{n}\left(\Delta t, t_{\mathrm{w}}\right) \sim f_{\Delta}(\alpha, \eta)$ with

$f_{\Delta}(\alpha, \eta)=\Phi(\eta) \Phi\left(\frac{\eta}{\sqrt{1+2 \alpha}}\right)-\left\{\frac{2}{\pi} \int_{0}^{\operatorname{arccot} \sqrt{\alpha}} \mathrm{d} z \mathrm{e}^{-\left[\eta^{2} /(1+\alpha)\right] \sec ^{2}(z)}\right\}^{2}$,

where $\sec (z)=1 / \cos (z)$. The scaling of the difference between the four-point susceptibilities $\Delta \chi\left(\Delta t, t_{\mathrm{w}}\right)=\chi\left(\Delta t, t_{\mathrm{w}}\right)-\mathcal{X}\left(\Delta t, t_{\mathrm{w}}\right)=\sum_{n} \Delta C_{n}\left(\Delta t, t_{\mathrm{w}}\right)$ then follows by analogy with (47): writing $\Delta \chi\left(\Delta t, t_{\mathrm{w}}\right) \sim \sqrt{t_{\mathrm{w}}} F_{\Delta}(\alpha)$ we obtain via integration of (55) over $\eta$,

$F_{\Delta}(\alpha) \sim \frac{4}{\sqrt{\pi}}\left\{\sqrt{1+2 \alpha}-1+\frac{4}{\pi}\left[\arctan \sqrt{2 \alpha}-\sqrt{\frac{1+\alpha}{2}} \arctan 2 \sqrt{\alpha(1+\alpha)}\right]\right\}$.

Plots of $f_{\Delta}(\alpha, \eta)$ and $F_{\Delta}(\alpha)$ are shown in Figure 4. Comparing the vertical scales in Figures 1 , 2, 4 demonstrates that the standard functions $C_{n}\left(\Delta t, t_{\mathrm{w}}\right)$ and $\chi\left(\Delta t, t_{\mathrm{w}}\right)$ are completely dominated by the two-spin contributions (55), (56). A plot of the fourpoint susceptibility $\chi_{n}\left(\Delta t, t_{\mathrm{w}}\right)=\mathcal{X}_{n}\left(\Delta t, t_{\mathrm{w}}\right)+\Delta \chi_{n}\left(\Delta t, t_{\mathrm{w}}\right) \sim \sqrt{t_{\mathrm{w}}}\left[F_{C}(\alpha)+F_{\Delta}(\alpha)\right]$, for instance, would be indistinguishable by eye from the inset of Figure 4 .

Therefore, as claimed, the standard four-point function (11) and its associated fourpoint susceptibility (31) are not suitable for measuring genuine four-point correlations in the coarsening dynamics of the Glauber-Ising chain. In comparison to strongly heterogeneous systems the relative magnitudes of, e.g., the connected four-point susceptibility (inset in Figure 22) and the corresponding two-point bias (inset in Figure 4) are reversed in coarsening systems.

\section{Conclusions}

In this paper we have explored dynamical heterogeneities in coarsening systems by studying multi-point correlations in the dynamics of the Glauber-Ising chain. Since conventional four-point correlation functions become dominated by strong spatial correlations that develop in coarsening systems at late times, we considered "connected" four-point functions where these uninteresting two-point contributions are eliminated. We were able to obtain exact results and scaling forms for these functions and the associated spatial integral, i.e. the connected four-point susceptibility. As a function of the time difference $\Delta t$, this multi-point susceptibility has an extremum, as is found in glass formers, for times of the order of the waiting time, indicating the timescale for which dynamic heterogeneity is maximal.

Interestingly, we found that the connected four-point susceptibility is negative throughout, and we were able to give an interpretation for this behaviour in terms 


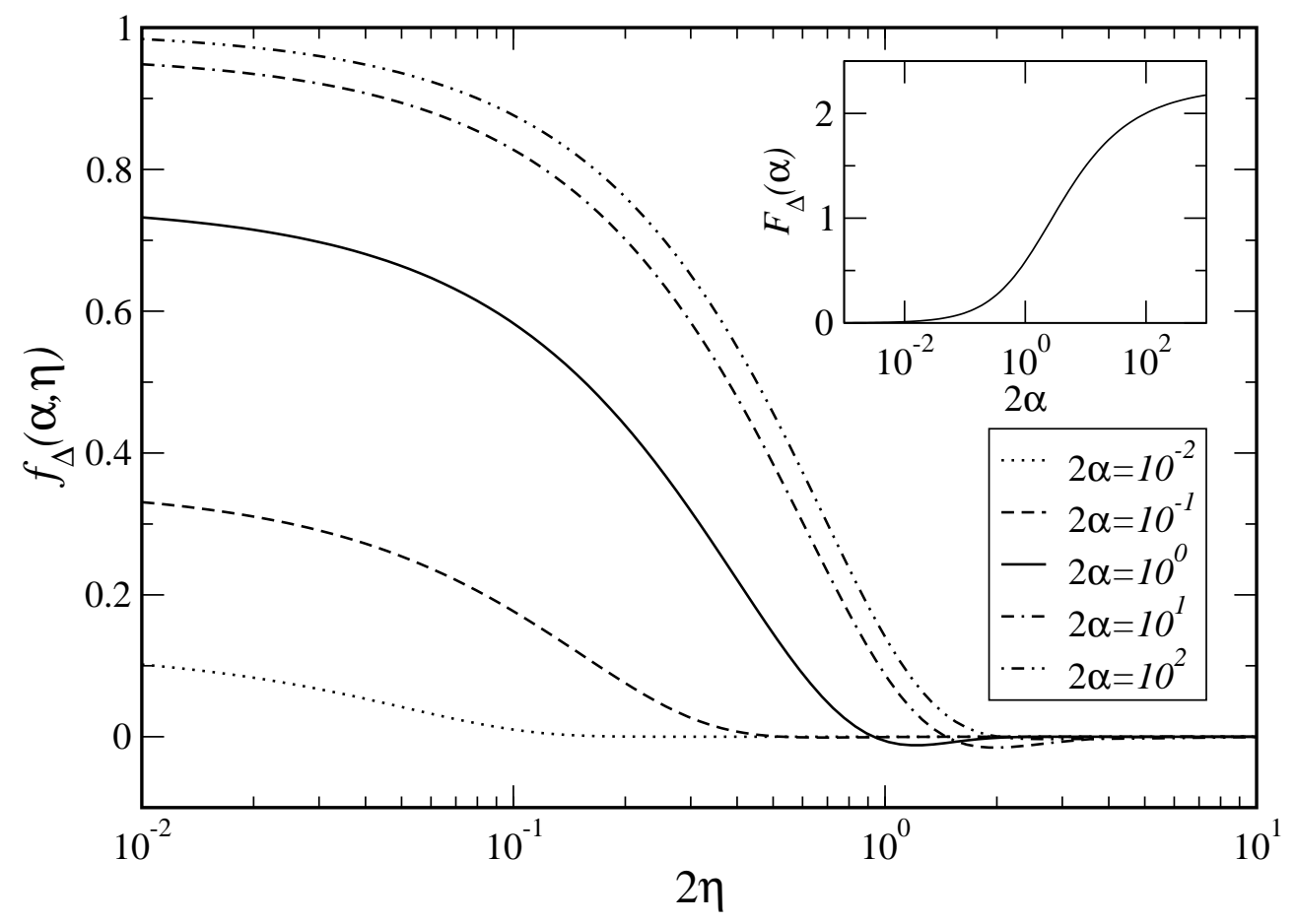

Figure 4. Plots of the scaling expansion (55) of $\Delta C_{n}\left(\Delta t, t_{\mathrm{w}}\right)$ versus scaled distance $2 \eta=|n| / \sqrt{t_{\mathrm{w}}}$ for various time ratios $2 \alpha=\Delta t / t_{\mathrm{w}}$. Inset: Normalized contribution $F_{\Delta}(\alpha)$ of the two-spin terms to the four-point susceptibility $\chi\left(\Delta t, t_{\mathrm{w}}\right)$, Equation (56).

of the dynamics of domain walls, which undergo free diffusion and pair annihilation. The negative sign of the susceptibility directly reflects the fact that there is an effective repulsion between the domain walls, each having a "depleted zone" around it where the likelihood of finding another domain wall is low. At equilibrium, on the other hand, domain wall positions are uncorrelated and this leads to the vanishing of the susceptibility, and of the underlying four-point correlations, for all $\Delta t$. This latter result, which we established using explicit expressions for four-spin correlations, appears rather non-trivial. It would be interesting to verify whether it also extends to equilibrium correlation functions of higher order. If it does, one suspects that there should be a deeper reason, possibly related to the mapping of the Glauber-Ising chain dynamics to free fermions [17].

We also discussed the spatial dependence of the connected four-point correlation functions. This has a richer structure than one might have expected, but the random walk picture again gave a good qualitative (and, in some regimes, quantitative) understanding of our exact results. In future work, it will be interesting to see if and how our findings generalize to other coarsening systems. Encouragingly, simulations show that many of the key features we found here extend at least to two-dimensional Ising models [13. 


\section{Acknowledgments}

We acknowledge financial support from the Austrian Academy of Sciences and EPSRC Grant No. 00800822 (PM), and EPSRC Grants No. GR/R83712/01, GR/S54074/01 and the University of Nottingham Grant No. FEF 3024 (JPG).

\section{References}

[1] M D Ediger. Spatially heterogeneous dynamics in supercooled liquids. Annu. Rev. Phys. Chem., 51:99-128, 2000.

[2] H Sillescu. Heterogeneity at the glass transition: a review. Journal of Non-Crystalline Solids, 243(2-3):81-108, 1999.

[3] S C Glotzer. Spatially heterogeneous dynamics in liquids: Insights from simulation. J. Non-Cryst. Solids, 274:342-355, 2000.

[4] G Diezemann. Dynamic heterogeneities in the out-of-equilibrium dynamics of simple spherical spin models. Phys. Rev. E, 68(2):021105, 2003.

[5] C Donati, S Franz, S C Glotzer, and G Parisi. Theory of non-linear susceptibility and correlation length in glasses and liquids. J. Non-Cryst. Solids, 307:215-224, 2002.

[6] S Franz, C Donati, G Parisi, and S C Glotzer. On dynamical correlations in supercooled liquids. Philos. Mag. B, 79(11-12):1827-1831, 1999.

[7] S Franz and G Parisi. On non-linear susceptibility in supercooled liquids. Journal of Physics: Condensed Matter, 12(29):6335-6342, 2000.

[8] J P Garrahan and D Chandler. Geometrical explanation and scaling of dynamical heterogeneities in glass forming systems. Phys. Rev. Lett., 89:035704, 2002.

[9] L Berthier. Finite-size scaling analysis of the glass transition. Phys. Rev. Lett., 91:055701, 2003.

[10] L Berthier and J P Garrahan. Real space origin of temperature crossovers in supercooled liquids. Phys. Rev. E, 68:041201, 2003.

[11] G Biroli and J P Bouchaud. Diverging length scale and upper critical dimension in the modecoupling theory of the glass transition. Europhys. Lett., 67:21-27, 2004.

[12] R L Jack, L Berthier, and J P Garrahan. Static and dynamic lengthscales in a simple glassy plaquette model. cond-mat/0502120, 2005.

[13] P Mayer, H Bissig, L Berthier, L Cipelletti, J P Garrahan, P Sollich, and V Trappe. Heterogeneous dynamics of coarsening systems. Phys. Rev. Lett., 93:115701, 2004.

[14] R J Glauber. Time-dependent statistics of the Ising model. J. Math. Phys., 4:294, 1963.

[15] P Mayer and P Sollich. General solutions for multispin two-time correlation and response functions in the Glauber-Ising chain. J. Phys. A: Math. Gen., 37(1):9-49, 2004.

[16] L S Gradshteyn and I M Ryzhik. Table of Integrals, Series, and Products. Academic Press, New York, 2000.

[17] B U Felderhof. Spin relaxation of the Ising chain. Rep. Math. Phys., 1:215-234, 1971.

[18] J E Santos. The duality relation between Glauber dynamics and the diffusion-annihilation model as a similarity transformation. J. Phys. A-Math. Gen., 30(9):3249-3259, 1997. 